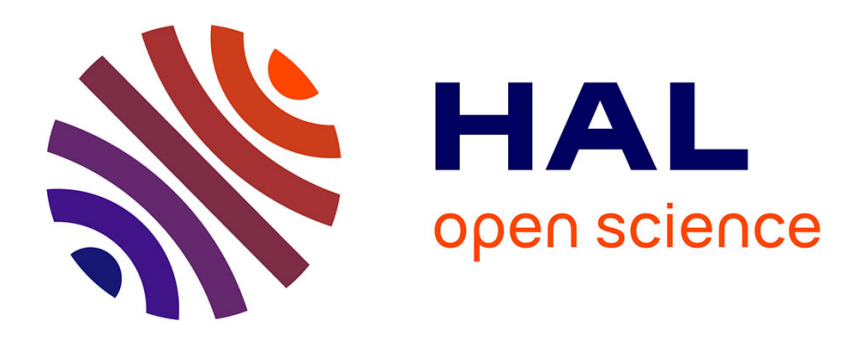

\title{
Pré/postpositions, verbes et aspect : trois isomorphismes remarquables en anglais, en français et en basque
}

Didier Bottineau

\section{To cite this version:}

Didier Bottineau. Pré/postpositions, verbes et aspect: trois isomorphismes remarquables en anglais, en français et en basque. Faits de langues, 2014, Prépositions et aspectualité, 44, pp.33-45. hal01816826

\section{HAL Id: hal-01816826 \\ https://hal.science/hal-01816826}

Submitted on 15 Jun 2018

HAL is a multi-disciplinary open access archive for the deposit and dissemination of scientific research documents, whether they are published or not. The documents may come from teaching and research institutions in France or abroad, or from public or private research centers.
L'archive ouverte pluridisciplinaire HAL, est destinée au dépôt et à la diffusion de documents scientifiques de niveau recherche, publiés ou non, émanant des établissements d'enseignement et de recherche français ou étrangers, des laboratoires publics ou privés. 


\title{
Pré/postpositions, verbes et aspect : trois isomorphismes remarquables en anglais, en français et en basque
}

\author{
Didier Bottineau*
}

\section{ProblematiQue}

Du côté du signifié, l'existence d'une dimension aspectuelle dans la sémantique des prépositions est un fait qui a retenu l'attention (Cadiot 2002) et constitue l'objet du présent volume. Du côté du signifiant, que cette sémantique puisse dans certains cas trouver son origine dans l'existence de marqueurs isomorphes ou transcatégoriels n'a pas fait l'objet d'une discussion approfondie. Les lignes qui suivent étudient trois cas de cet ordre :

- en basque, un contraste morphématique $k / n$ se trouve impliqué dans un nombre important de microsystèmes grammaticaux, dont celui des postpositions spatiales et relationnelles correspondant à de et en et celui des participes verbaux prospectifs et «inspectifs » correspondant mutatis mutandis à l'infinitif et au participe présent romans ;

- en français et en anglais, on observe un isomorphisme phonologique total ou partiel liant les prépositions aux flexions aspectuelles verbales, et ce en dépit d'origines diachroniques hétérogènes : français en (préposition et clitique) et -ant, anglais in et -ing.

L'approche sera guidée par la Théorie des actes corpori-mentaux langagiers (TACML) dans le paradigme des linguistiques enactives (Bottineau 2010a, 2013). La linguistique enactive est un constructivisme qui envisage la parole humaine comme l'exercice incarné individuel ou dialogal d'une forme et technique particulière de l'intelligence sociale. Celle-ci consiste en l'autodétermination d'actes de conscience par un contrôle vocal outrepassant la perception immédiate en mobilisant des processus signifiants normatifs qui restaurent et recombinent des représentations construites dans un ensemble d'interactions formant une chaîne dialogique. Dans ce cadre, l'expérience du signifiant dans sa dimension motrice et sensorielle, individuelle et interactive, joue un rôle constituant dans le processus d'accès conjoint à des états de conscience et de connaissance : la parole n'est pas la trace ou l'encodage du fait cognitif langagier, mais son vecteur indissociable (ce qui n'exclut pas l'existence

* CNRS, MoDyCo, Université Paris Ouest, Courriel : didier.bottineau@u-paris10.fr 
d'autres vecteurs de cognition que la phonation); elle permet aussi bien de coordonner la réflexion d'autrui avec la sienne propre (parole adressée, exophasie) que de stimuler la cogitation personnelle (endophasie, simulation réflexive de parole intérieure). L'approche enactive est en partie compatible avec les théories de l'énonciation et de la cognition par son constructivisme, le rejet du référentialisme iconique, l'auto-organisation des systèmes métalinguistiques, mais elle en diffère par l'embodiment radical au niveau même du signifiant (inscription corporelle de l'émergence de l'esprit) : elle relève du paradigme de la cognition dite des quatre $\mathrm{E}$ (embodied, embedded, enactive, extended) (Menary 2010), distribuée et dialogique, issue de la phénoménologie merleaupontyenne du corps via la théorie de l'autopoï̀se et de l'enaction de Varela et Maturana (Varela et al. 1991).

Dans cette étude, sans entrer dans les détails de la submorphémie (théorie des cognèmes), on applique ces principes aux marqueurs donnés en titre, et on fait l'hypothèse que ces trois isomorphismes parallèles sont la signature d'une valeur instructionnelle interprétative commune aux relations spatiales (prépositions / postpositions selon le type de langue) et au profilage de l'aspect (flexions et affixes verbaux), intégrée conjointement à différentes parties du système grammatical. On repose ainsi la question de l'invariance dans des termes différents de ceux des nombreuses études antérieures sur la question (pour en, Gaatone 1980, Cervoni 1991, Franckel \& Lebaud 1991, Achard-Bayle 2005).

\section{LE SYSTEME $K / N$ ET LA TRANSCATEGORIALITE EN BASQUE}

Pour notre propos, les caractéristiques pertinentes du basque sont les suivantes :

- tout syntagme nominal, verbal ou propositionnel (dans le cas des subordonnées) est clôturé à droite par un train de marqueurs grammaticaux agglutinés, une chaîne de postpositions qui confère à l'élément qui précède divers paramètres tels que la définitude, le nombre, la relation syntaxique à un autre syntagme : etxeko atea 'porte-de maisonla' $\rightarrow$ « la porte de la maison ».

- chaque postposition est «transcatégorielle» (Bottineau 2009), affixable à la dernière unité lexicale de syntagmes de natures diverses : appliqué à un $\mathrm{SN}, k o$ produit habituellement un $\mathrm{SN}$ adjectivé muni d'une valeur délimitative correspondant à celle de la préposition de; appliqué à un participe accompli comme ikusi «voir» (perfectif) / «vu», ko livre un participe prospectif ikusiko 'voir-de' $\rightarrow$ «à voir» (glose) correspondant approximativement à l'infinitif et permettant la formation du futur en combinaison avec l'auxiliaire : ikusiko duzu 'voir-de le-vous' $\rightarrow$ « vous le verrez ».

- chaque postposition peut elle-même être analysée comme une composition d'éléments formateurs : à côté de ko du participe futur se trouve ke de la conjugaison de l'auxiliaire aux formes hypothétiques (potentiel, conditionnel) : ikusi nahi nuke 'voir volonté je-le-cond' "j'aimerais le voir »; et face à ke se trouve justement $-n$ pour le passé des auxiliaires et 
Pré/postpositions, verbes et aspect : trois isomorphismes remarquables en anglais, en français et en basque

verbes conjugués (ikusi nuen 'voir le-je-pass' $\rightarrow$ je le vis »). En analyse distributionnelle, on observe des paires de marqueurs tels que ko / $-(e) n$ (les deux génitifs délimitatif et possessif), $k a$ / $n a$ (les marques de conjugaison de la $2^{\mathrm{e}}$ personne tutoyée masculine et féminine), -ago / -en (les degrés de l'adjectif : comparatif et superlatif) et bien d'autres. Si l'on considère l'ensemble de ces marqueurs comme des composés formés de plusieurs éléments formateurs, alors on constate que l'ensemble de ces contrastes représentent une série d'occurrences de la paire fondamentale $k / n$ diversement intégrée dans des opérateurs utilisés dans des microsystèmes diversifiés, comme si cette paire avait "métastasé » les différents microsystèmes de la grammaire basque en général. Le tableau infra donne une vue d'ensemble de cette diffusion. Tous les rapprochements indiqués ne font pas l'objet d'une égale robustesse en matière de validation et on ne peut ici en discuter le détail ; le point important pour cette étude-ci est que dans le cas des génitifs et des participes aspectuels, l'origine diachronique commune des deux contrastes est établie, mentionnée dans les grandes grammaires du basque, et peut être considérée comme un fait :

\begin{tabular}{|c|c|c|}
\hline & \multicolumn{2}{|c|}{ Relateurs de dépendance } \\
\hline & De départ : K (opératif) & D'arrivée : N (résultatif) \\
\hline $\begin{array}{l}\text { Cas « nominaux » } \\
{[\mathrm{SN}]-\mathrm{X}} \\
\text { (postpositions } \\
\text { spatiales et } \\
\text { relationnelles de } \\
\text { portée } \\
\text { syntagmatique) }\end{array}$ & $\begin{array}{l}\text { aitak « papa » (ergatif) } \\
\text { Aitak liburua irakurtzen du } \\
\text { "papa-ERG livre-DEF lisant le-il } \\
\text { "papa lit le livre » } \\
\text { etxeko « de la maison »: Locatif } \\
\text { conceptuel } \\
\text { Etxeko atea 'maison-de porte-la' } \\
\text { «la porte de la maison» }\end{array}$ & $\begin{array}{l}\text { etxean « dans la maison» (locatif) } \\
\text { inessif / locatif environnemental } \\
\text { aitaren « de papa » : possessif / } \\
\text { locatif personnel } \\
\text { aitaren kapela 'papa-le-de chapeau- } \\
\text { le' « le chapeau de papa» }\end{array}$ \\
\hline $\begin{array}{l}\text { Participes } \\
\text { «futurs » } \\
{[\mathrm{V}]-\mathrm{X}} \\
\text { Rad. } V+\text { postp. }= \\
\text { part. prosp. }\end{array}$ & $\begin{array}{l}\text { ikusiko } \\
\text { Participe prospectif } \\
\text { Ikusiko duzu 'voir-de le-vous' « vous } \\
\text { verrez » (catégorique) }\end{array}$ & $\begin{array}{l}\text { ikusiren } \\
\text { Participe prospectif } \\
\text { Ikusiren duzu 'voir-de le-vous' } \\
\text { «vous verrez » (préconstruit) }\end{array}$ \\
\hline Temps verbaux & $\begin{array}{l}\text { Hypothétique : V/Aux-ke } \\
\text { Ikusiko luke 'voir-de le-(il)-HYP' } \\
\text { « il le verrait » }\end{array}$ & $\begin{array}{l}\text { Passé : V/Aux-n } \\
\text { Ikusi zuen 'vu le-(il)-PASSE' } \\
\text { « il le vit » }\end{array}$ \\
\hline $\begin{array}{l}\text { Nom (dé)verbal } \\
{[\mathrm{V}]-t z e-\mathrm{X}} \\
{[\mathrm{Rad} . \mathrm{V}+t z e]-\mathrm{X}}\end{array}$ & $\begin{array}{l}\text { ikusteko 'regard-age-de', «à } \\
\text { regarder » } \\
\text { Zer da ikusteko ? } \\
\text { 'Qui est voir-de' } \\
\text { "Qu'y a-t-il à voir ? " } \\
\text { Nire amak buruan musu bat eman } \\
\text { eta lo egiteko esan zidan. } \\
\text { "Ma mère me donna un baiser sur la } \\
\text { tête et me dit de dormir » }\end{array}$ & $\begin{array}{l}\text { ikusten 'regard-age-en', } \\
\text { "regardant " } \\
\text { Nire leihotik ikusten dut } \\
\text { 'moi-de fenêtre-par le-je' } \\
\text { « je regarde par ma fenêtre » } \\
\text { Ikustean 'regard-age-le-en/dans', } \\
\text { " (en) regardant » } \\
\text { Hori ikustean } \\
\text { 'cela 'regard-age-le-en/dans' }\end{array}$ \\
\hline
\end{tabular}




\begin{tabular}{|c|c|c|}
\hline & & «En regardant (voyant) cela » \\
\hline $\begin{array}{l}\text { Degrés de } \\
\text { l'adjectif }\end{array}$ & $\begin{array}{l}\text { Comparatif : } \\
\text { ederrago 'beau-DEF-ko } \backslash \text { go' } \\
\text { " plus beau » } \\
\text { Zure etxea nirea ederragoa da } \\
\text { 'Vous-de maison-la moi-de-la beau- } \\
\text { DEF-de-DEF est' } \\
\text { "Votre maison est plus belle que la } \\
\text { mienne» }\end{array}$ & $\begin{array}{l}\text { Superlatif : } \\
\text { ederren }(\text { a }) \text { 'beau-de' } \\
\text { «(le) plus beau » } \\
\text { Zure etxea ederrena da } \\
\text { 'vous-de maison-DEF beau-de-DEF est' } \\
\text { « votre maison est la plus belle» }\end{array}$ \\
\hline $\begin{array}{l}\text { Conjugaisons : } \\
2^{\mathrm{e}} \text { personne } \\
\text { tutoyée }\end{array}$ & $\begin{array}{l}\text { Masculine : }-\boldsymbol{k a} \boldsymbol{a}-(-\boldsymbol{a}-,-\boldsymbol{k}) \\
\text { Egia duk 'vrai-DEF le-tu' } \\
\text { «tu as raison » }\end{array}$ & $\begin{array}{l}\text { Féminine : }- \text { na- }(-n) \\
\text { Egia dun 'vrai-DEF le-tu' } \\
\text { «tu as raison » }\end{array}$ \\
\hline Subordination & $\begin{array}{l}\text { Injonctive : génitif conceptuel du } \\
\text { nom verbal (inactuel) } \\
\text {... eta lo egiteko esan zidan. } \\
\text { «... et il me dit de dormir » } \\
\text { Causale : génitif propositionnel } \\
\text { polyphonique (actuel) } \\
\text { Egia da. Egia delako. } \\
\text { 'Vrai-DEF est. [Vrai-DEF est]que-est- } \\
\text { de.' } \\
\text { "C'est vrai ». « Parce que c'est } \\
\text { vrai. » }\end{array}$ & $\begin{array}{l}\text { Interrogative et relative : génitif } \\
\text { possessif propositionnel, non } \\
\text { polyphonique } \\
\text { Ezagutzen dugun emakumea etorriko } \\
d a \\
\text { '[connaissant la-nous] }] \text { de femme-DEF } \\
\text { venir-de est' } \\
\text { «La femme que nous connaissons } \\
\text { viendra. » } \\
\text { (relatif = prédication inaccomplie : + } \\
\text { "postcédant » à droite) } \\
\text { Ez dakit etorriko den } \\
\text { 'NEG sais venir-de est-de' « Je ne sais } \\
\text { pas s'il viendra » } \\
\text { (Aux da }+ \text { interrogatif }-n=\text { den }= \\
\text { assertion inaccomplie) }\end{array}$ \\
\hline
\end{tabular}

Dans les grandes lignes, on peut esquisser l'invariant du contraste d'éléments formateurs $k / n$ comme suit: $k$ opère une saisie aspectuelle inchoative, prospective et non présupposante du processus sémantique traité par l'opérateur intégrant appliqué au domaine grammatical qui le concerne, alors que $n$ réalise une saisie aspectuelle cursive, «inspective» et présupposante du processus correspondant. Ce contraste procédural aspectualise le processus de formation du sens et affecte comme suit divers microsystèmes :

- cas des génitifs: -ko s'applique à la notion lexicale de langue non déterminée (cf. etxeko atea) et réalise un repérage de principe de $\mathrm{N} 2$ par rapport à N1 pris comme notion de langue ; -en s'applique à un SN1 de discours, le plus souvent déterminé, si bien qu'il profile une relation de possession par un possesseur effectivement repéré et défini, muni de propriétés présupposées (l'animation, le statut social, ou autres selon les contextes. - $k o$ s'adosse à $\mathrm{N} 1$ pris comme repère primitif et préconstruit lexical de langue, alors que -en s'applique à une notion retraitée en discours, signant un degré plus avancé de la construction de la relation.

- cas des participes futurs (pour le détail cf. Bottineau 2008) : pour les locuteurs qui effectivement alternent les deux formes en $-k o$ et $-e n$ des 
Pré/postpositions, verbes et aspect : trois isomorphismes remarquables en anglais, en français et en basque

participes futurs, le premier est simplement prospectif et non présupposant, alors que le second exprime une prédiction ou décision fondée sur des motivations antérieures (ikusiko / ikusiren duzu «vous verrez (bien) »).

- participe présent (ikusten 'voir-NOM verBaL-INESS' $\rightarrow$ «dans le voir», «voyant »: le nom verbal est porté à l'inessif locatif $-n$ (marqué par le même élément formateur $n$ que le génitif possessif), ce qui fait littéralement de l'inaccompli cursif une localisation «dans » le déroulement du procès désigné par le verbe. On trouve également une forme ikusteko 'voir- ${ }^{-}{ }^{-}{ }_{\mathrm{GEN}}$ ' " de voir » utilisée comme "subordonnée infinitive » après les verbes volitifs : Egia esateko esan dut 'vérité-la dire$\mathrm{NV}^{-} \mathrm{GEN}$ dire le-lui-je' $\rightarrow$ « je lui ai dit de dire la vérité ».

- conjugaisons et déflexivité en basque : le basque ancien ne possédait qu'une «conjugaison synthétique » par laquelle tous les marqueurs de personne, temps et modalité s'agglutinaient directement au radical verbal, ne laissant aucune place pour des flexions d'aspect : (ba)daramakiot 'leapporter-à-lui-je' $\rightarrow$ « je le lui apporte », (ba)neramakion 'je-le-apporter-à lui-pass, $\rightarrow$ «je le lui apportai ». En basque actuel, seuls une douzaine de verbes courants ont conservé ce modèle agglutinatif (dont eraman «apporter»), alors que tous les verbes présentent une conjugaison périphrastique de la forme participe + auxiliaire : le participe reçoit les spécifications d'aspect par les postpositions génitives (participe «passé » eraman, participe présent $=$ nom verbal à l'inessif $=$ eramaten, participes futurs = participe passé / génitif eramango / eramanen $)$; l'auxiliaire final reçoit les spécifications de personne, temps et modalité, délocalisées par déflexivité progressive (Bottineau 2010b) : eramango diot « je le lui apporterai », eramango nion « je le lui apporterais »(futur dans le passé). En vertu de la déflexivité, le contraste temps / mode au niveau de l'auxiliaire (passé - $n$, hypothétique -ke) et les contrastes aspectuels au niveau du participe (inaccompli $-n$, à accomplir -ko / -en) font systématiquement appel à l'unique paire de base $k / n$, comme on peut le constater.

- degrés de l'adjectif (handiago «plus grand», handien « le plus grand ») : -ko (voisé en -go) repère le nom par rapport à une propriété handi «grand» déjà prédiquée d'une autre unité (handi-a) ('Jean est de le grand' = 'Jean est grand par rapport à ce dont on sait déjà que c'est grand' $=$ 'Jean est plus grand que l'occurrence déjà considérée'). Pour le superlatif, $-n$ repère Jean par rapport à la propriété indéterminée (handien : pas de $a$ ) et applicable à toute autre occurrence envisageable, ce qui fait de Jean « le plus grand » de tous. Le contraste comparatif / superlatif s'obtient en faisant varier l'étendue du standard laissé en arrière-plan, spécifique ou générique, et cette variation s'obtient par le jeu des niveaux aspectuels de construction de la relation: incipiente avec -ago, plus avancée avec -en.

- formes conjuguées du verbe et de l'auxiliaire : l'hypothétique -ke est clairement prospectif et ouvert sur une actualisation possible à venir, alors 
que le passé - $n$ repère un procès spatialement comme un « là » ou «ça » détenu par la mémoire (le passé verbal $-n$ étant aussi isomorphe de l'inessif-locatif $-n$ : etxean 'maison-la-à' $\rightarrow$ « à la maison ».

On constate que plusieurs microsystèmes grammaticaux spécialisés se construisent autour d'un mécanisme général commun d'aspectualisation $\mathrm{du}$ processus traité, selon le contraste inchoation / déroulement. Ce mécanisme s'applique à l'aspect verbal (participes, noms verbaux), mais aussi à des processus plus abstraits tels que le repérage de la propriété d'une entité par rapport à une autre entité qui en est munie (comparatif) ou à une classe d'unité (superlatif) ; ou encore, au repérage simple d'une unité par rapport à une autre, en tant que notion de langue (génitif en -ko) ou occurrence de discours (génitif en -en). La transcatégorialité des opérateurs permet l'application de «logiciels mentaux » uniques à des domaines d'application diversifiés, et la compositionnalité même de ces opérateurs, typologiquement caractéristique de l'agglutination, permet à des couples d'éléments formateurs radicaux de figurer dans toute la grammaire de manière récurrente. Derrière la productivité quantitative et la diversité qualitative de la morphosémantique basque, se cachent des principes fondamentalement simples qui expliquent l'ergonomie cognitive du système, sa maniabilité pour ses utilisateurs, et son acquérabilité en dépit de sa complexité apparente - une illustration de la propriété des systèmes dynamiques vivants que Berthoz 2009 nomme simplexité. Dans cette architecture, les marqueurs verbaux et les postpositions de relations syntaxiques sont traités par un appareil économique d'opérateurs d'aspectualisation qui confèrent un air de famille aux fonctionnements des différentes zones du système.

Certaines approches comme les travaux de Toussaint sur la motivation cognitive de l'articulation des signifiants et la théorie des cognèmes (Bottineau 2003) suggèrent que l'invariant de contrastes radicaux tels que $k / n$ en basque se fondent sur la proprioception et semiosis des propriétés motrices articulatoires mêmes des phonèmes concernés $(/ \mathrm{k} /$ est « d'arrière », précoce dans la cavité buccale envisagée dans le sens de l'expiration; /n/ apical, "d'avant», est en réalité tardif, et englobant du fait de la résonance nasale). On laisse ici de côté cette facette du problème, suggérant simplement le fait que le basque mobilise très largement cette tendance au réinvestissement cognitif de l'expérience de l'articulation des sons langagiers dans le cadre des oppositions mises en évidence par les microsystèmes grammaticaux.

\section{ANGLAIS ET FRANÇAIS}

Là où le basque utilise les mêmes éléments formateurs $k$ et $n$ pour la constitution parallèle des postpositions et des morphèmes verbaux, l'anglais et le français marquent en parallèle la notion d'intériorité spatiale et de déroulement cursif par des opérateurs quasi isomorphes :

\begin{tabular}{|l|l|l|}
\hline Prép. $+\mathbf{N}$ & V-flexion & Prép. + V-flexion \\
\hline in $+\mathrm{N}$ in an attempt to & V-ing attempting to & in + V-ing in saying this \\
\hline en $+\mathrm{N}$ en marche & V-ant marchant & en $+\mathrm{V}$-ant en disant cela \\
\hline
\end{tabular}


Pré/postpositions, verbes et aspect : trois isomorphismes remarquables en anglais, en français et en basque

Il ne s'agit pas de traiter in et -ing ou en et -ant comme marqueurs uniques et transcatégoriels, mais de rendre compte de la «communauté d'esprit» qui a amené ces opérateurs à un amalgame morphosémantique avancé.

\subsection{Anglais}

La valeur instructionnelle de la préposition in consiste à faire repérer une entité relativement à un espace confiné ou délimité : the bird is in the cage «l'oiseau est dans la cage ». La diversité des valeurs d'emploi ${ }^{1}$ tient à ce que la nature de l'espace visé varie de l'objet concret aux loci les plus abstraits tels que des espaces discursifs et mentaux, domaines thématiques, points de vues, manières de traiter un problème, le cadrer, l'évaluer : in principle / reality / short I a nutshell, in a decisive move. Les situations envisagées sont hétérogènes, mais le processus mental de construction du sens, considéré dans sa dynamique procédurale, est le même : de même que in fait repenser l'entité the bird dans le cadre d'un domaine matériel spécifié par the cage, in fait repenser une relation prédicative dans le cadre d'un domaine d'interprétation spécifié par un nom abstrait comme reality ou dans celui d'un mode énonciatif spécifié par short ou $a$ nutshell, l'un et l'autre impliquant topologiquement les limites et le confinement requis pour fournir un domaine ciblé compatible avec l'opérateur in (de même que reality et principle s'opposent à tout ce qui leur est extérieur et se comprennent comme des domaines d'acceptabilité confinés).

Pour sa part, l'opérateur -ing «représente une vision sécante du temps d'évènement » selon la doxa guillaumienne, ou, plus exactement, opère une saisie sécante récurrente du temps d'évènement (Bottineau 2001), articulant un échantillonnage cursif par la répétition de la saisie et la mise en valeur des segments dissociés : -ing donne à l'interprétant l'instruction de se figurer une séquence intermédiaire d'un déroulement compris entre deux zones aveugles, l'accompli et l'inaccompli, eux-mêmes schématiquement limités par des bornes initiale et finale (qu'elles soient instanciées ou non dans le cas du procès envisagé en fonction de l'aspect lexical du verbe, sa télicité, sa télicisation par la complémentation actancielle et/ou circonstancielle, etc.). En d'autres termes, -ing demande à l'interprétant de prélever un échantillon cursif de l'actualisation du procès, d'extraire un quantum processuel symptomatique du fonctionnement d'un tout dynamique, et de l'observer analytiquement en vue d'en tirer un diagnostic. Ceci explique l'incompatibilité de -ing avec un sujet, lequel est par définition en situation de contrôle de l'actualisation de l'ensemble du temps d'évènement, sauf si ce contrôle est restreint par l'auxiliaire be à l'extension de l'échantillon focalisé par -ing, ce qui donne la forme dite progressive, utilisée lorsque le locuteur focalise l'attention de l'allocutaire sur un procès dont l'existence, la

\footnotetext{
${ }^{1}$ Dans la grammaire anglaise, in est traditionnellement traité comme un relateur spatial (Guimier 2007). Cette approche est contestée en particulier en théorie des opérations énonciatives, qui récuse la primauté de la valeur spatiale et les dérivations métaphoriques au profit d'une forme schématique abstraite (Gilbert 2004) ou topologique (Dufaye 2006).
} 
pertinence ou le sens avaient échappé : l'échantillonnage du déroulement et l'occultation en zone aveugle des segments accompli et inaccompli rend nécessaire des projections interprétatives rétrospective et prospective de nature à porter l'attention sur les présupposés et les implicatures, les tenants et aboutissants nécessaires à la compréhension de l'évènement; et la mise en avant du déroulement du procès est porteuse du déroulement même de l'interprétation vectorisé par ces projections, ce qui fait de -ing un opérateur relationnel et phénoménologique co-indexant la durée objective du procès (en $3^{\mathrm{e}}$ personne), la durée subjective de l'interprétation (en $2^{\mathrm{e}}$ personne) et la durée intersubjective de l'interlocution (adressée en $1^{\mathrm{e}}$ personne).

Hors de la «forme progressive», en l'absence de be, le sujet-contrôle est prédéfini, explicitement («me attempting ») ou implicitement (tous les autres) :

(1) Avoiding slouching is without doubt one of the simplest means to gain height naturally. Avoid looking like you're trying hard to look taller by wearing something that blends into your overall outfit. Avoid loud bold stripes that scream have a look at me attempting to look taller, with the intention that it doesn't become obvious that's the main reason why you're wearing clothes with vertical stripes. Wear something that blends a bit more into your overall outfit. (Article Trader, "How to increase my hight", 8 mai 2010) ${ }^{2}$

Ce qui est particulièrement intéressant, c'est qu'une expression comme attempting to peut être formulée par une locution prépositionnelle, in an attempt to, comme dans l'exemple suivant :

(2) Sarko standing on his toes, in an attempt to look taller.

(2') Sarko standing on his toes, attempting to look taller.

Dans (2), commentaire d'une photo par un blogueur ${ }^{3}$, le locuteur introduit le procès nominal au moyen d'une préposition, rhématisant le cadre d'interprétation attempt par rapport à l'opérateur d'intégration in qui l'introduit: le locuteur fournit personnellement un diagnostic et ne laisse pas entendre que le lecteur pouvait avoir deviné ou se faire une idée préalable de la signification du geste. Dans la reformulation (2'), -ing focalise un segment de déroulement du cadre interprétatif attempt, qu'il thématise par suffixation: l'effet de sens est que l'interprétation fournie par le locuteur était cette fois prévisible, éventuellement déjà produite par le lecteur, et correspondant à un faisceau de caractéristiques supposé connu du sujet «Sarko». Les deux formulations sont également recevables, mais correspondent à des mises en scènes divergentes du rapport interlocutif, l'une se fondant sur l'absence d'un référentiel commun, l'autre sur sa présence. D'un point de vue syntaxique, cette analyse implique que in et -ing représentent la même instruction métalinguistique, celle qui consiste à intégrer

2 http://www.articletrader.com/health/fitness/how-to-increase-my-height-its-amazinghow-these-simple-tips-will-help.html

${ }^{3}$ https://www.flickr.com/groups/62226056@N00/pool/page4/ 
Pré/postpositions, verbes et aspect : trois isomorphismes remarquables en anglais, en français et en basque

une entité à un cadre : avec in prépositionnel, l'opération d'intégration est prise en charge en temps réel au moment où cet opérateur est énoncé en syntaxe linéaire ; le cadre est visé prospectivement est introduit, présenté, annoncé et rhématisée, ce qui confère au tour une valeur heuristique. Avec-ing, l'opération d'intégration du cadre, amalgamée à ce cadre, est préconstruite et présupposée, ce qui a pour effet de thématiser l'ensemble et de le faire figurer comme l'objet possible d'une connaissance partagée dans l'interlocution, ou du moins disponible au sein de la communauté parlante de par les propriétés réputées établies du sujet. En d'autres termes, in et -ing correspondent aux versions déflexive et inflexive du même opérateur avec la même valeur instructionnelle : la même forme schématique dynamique est réalisée en des moments distincts de la linéarité selon que le locuteur cherche à la faire inventer ou retrouver par l'interprétant. Dans ces conditions, même si in et -ing sont distributionnellement des opérateurs de classes distinctes, ils opèrent fonctionnellement en suivant le même schéma d'action mentale, ce qui explique l'isologie aspectuelle liant la préposition et la flexion verbale.

On trouve enfin la construction en in $+\mathrm{V}$-ing, où le même acte de cadrage est réalisé à deux reprises complémentaires, avec deux portées syntaxiques et sémantiques distinctes :

(3) In attempting to sign up, my username was okay but my email address was listed as already used. Now I can't use either.

- la préposition in amorce l'opération de cadrage en site introductif et rhématise le cadre visé en vue de le présenter au lecteur;

- $\quad$ la flexion -ing fait de la tentative un cadre présupposé renvoyé à un sujetcontrôle prédéfini, en l'occurrence le sujet énonçant, intégré au possessif my (malgré la rupture de construction) et spécifié dans la seconde phrase $(I)$.

Cette structure à double cadrage est fréquemment utilisée avec les verbes déclaratifs (in saying this) lorsqu'il s'agit de réintroduire le déjà dit de l'allocutaire ou d'un tiers pour en présenter une réinterprétation novatrice ou en rupture par rapport aux présupposés. Dans le cas des énoncés (2) et (2'), in et ing s'excluent mutuellement parce qu'ils correspondent à deux moments de réalisation de la même opération de cadrage en s'opposant sur le critère de la rhématisation vs thématisation selon l'effet recherché par le locuteur en matière de convergence / divergence des points de vue dans l'interlocution selon la théorie de l'esprit intuitive qu'il se fait de l'allocutaire. En (3) ces deux opérateurs sont complémentaires parce qu'appliqués à des composantes distinctes de l'élaboration du sens: l'introduction d'un prédicat pour in et la préconstruction du sujet de ce même prédicat pour -ing, sans contradiction, sans collision entre coordinations interlocutives adverses.

\subsection{Français}


La valeur instructionnelle de la flexion -ant est schématiquement analogue à celle de -ing même si son spectre d'application syntaxique est très différent pour des raisons diachroniques de natures diverses (amalgame d'un suffixe nominal et d'une flexion verbale pour -ing, origine verbale et adjectivale de -ant) : focalisation d'un échantillon intermédiaire de déroulement et d'actualisation du procès. Pour sa part, la préposition en, au statut morphosémantique et syntaxique très controversé dans la littérature, est connue pour sa «valeur situative » : elle relie une entité de nature variable (personne, segment de discours) à un cadre concret ou abstrait et modifie transitoirement les propriétés de l'entité dans les limites de sa relation au cadre - ce qui revient à dire que là où -ant aspectualise la relation d'un sujet présupposé à un procès préconstruit, en aspectualise la relation d'une entité source à un cadre cible, et se présente iconiquement comme opérateur intermédiaire et relationnel libre sur le site syntaxique même de la mise en rapport (Bottineau 2013): J'ai passé le weekend dans les Cévennes $=$ le locuteur repère géographiquement «je » par rapport aux Cévennes, sans plus ; J'ai passé le weekend en Cévennes (formule courante dans le Gard) = les propriétés du sujet «je » sont transitoirement modifiées en fonction de ce que l'on connaît stéréotypiquement des Cévennes (lieu de repos, de randonnée, de truffières, de méditation, de résistance politique et religieuse, d'expérimentation écologique et altermondialiste...) ; dans la seconde formulation, le choix de en souligne une motivation du sujet et focalise la manière dont ce choix a pu l'affecter positivement dans sa réalisation :

(4) Et les premiers qui rouspètent quand ils vont se promener en Cévennes et que le portable ne passe pas !!

Hénault 2006 détaille sur corpus les conditions de ce type d'opposition en insistant sur le figement lié à l'emploi de en, le rôle de certaines extensions (adjectivales et autres) qui favorisent l'emploi de dans, et surtout les corrélations avec les temps verbaux (la mise à distance au passé simple favorise en, contrairement au passé composé et au présent).

De manière générale, en prépositionnel aspectualise la connexion d'une entité à une propriété adjectivale ou adverbiale, reliée par une connexion de type «incidence externe»: un homme en colère (mutation transitoire de «un homme » par la notion «colère » dans le cadre de la connexion en, ici adjectivale) ; en principe, tu as raison (mutation du statut assertif de «tu as raison » par le cadrage «principe » dans la saisie aspectuelle de la déclaration en cours, prise en charge par le locuteur). Les autres occurrences de en fonctionnent de la même manière : elles aspectualisent la relation syntaxique correspondant au site de leur implantation dans la linéarité de l'énoncé. Concrètement :

- cas du gérondif, Je m'arrêterai à la pharmacie en rentrant: dans cette position de ligateur coprédicatif, en acquiert certaines des propriétés

\footnotetext{
${ }^{4}$ Commentaire d'un blogueur relatif à une association qui s'oppose à l'installation d'un relais de téléphonie portable sur le toit d'une école à Nîmes :

http://www.midilibre.fr/2012/01/26/dma-ils-s-opposent-a-une-troisieme-antenne-relaisau-dessus-de-1-ecole,449058.php
} 
Pré/postpositions, verbes et aspect : trois isomorphismes remarquables en anglais, en français et en basque

syntaxiques d'un sujet (Bottineau 2013), de même que -ant occupe le site de la flexion d'accord verbal en rang personnel. Tout se passe comme si la corrélation en / -ant retraitait en bloc les termes indissociables d'une relation prédicative soudée par l'accord en rang personnel, à savoir un couple sujet / flexion verbale, dans le cadre d'une ligature coprédicative. En et -ant remplacent respectivement les marqueurs du sujet et de la flexion par des marqueurs aspectuels hétérographes du fait de leurs appartenances catégorielles propres mais homophones du fait de l'unité de traitement aspectuel métalinguistique qu'ils signifient conjointement, à savoir la transition d'une relation à l'autre, avec mise en devenir des deux pôles de la relation. S'appuyant sur le sujet prédéfini je de $m$ 'arrêterai, en, marque de devenir précédant rentrant, réinscrit ce sujet acquis dans le devenir exprimé par le -ant du temps d'évènement verbal d'une prédication seconde, ce qui revient à présenter comme synchronisés en parallèle les traitements des deux sujets, le vrai «je » et son avatar en, et les traitements des deux temps d'évènements, $m$ 'arrêterai et rentrant. En préverbal du gérondif inscrit un opérateur instruisant un déroulement appliqué à la position fonctionnelle du sujet et signifie, littéralement, «sujet en retraitement», «sujet dont la conceptualisation est déjà engagée » pour le locuteur, ce qui lui confère une valeur aspectuelle purement métalinguistique, dégagée de toute référence à "l'extralinguistique », mais en prise avec la chronologie mentale du traitement cognitif de la prédication par le locuteur. Lorsque le gérondif suit la principale, le fonctionnement est le même pour l'allocutaire, qui dispose rétroactivement du sujet courant auquel en supplée. Lorsque le gérondif est utilisé en introduction (En passant par la Lorraine avec mes sabots), seul le locuteur sait à quel sujet courant supplée en, alors que l'allocutaire le reçoit comme instruction d'attente, annonce d'un sujet courant rhématisé. Le fait que en gérondival retraite un sujet stabilisé par une marque de devenir appliquée à la fonction traitée en cette position de ligature est illustré par l'ensemble des combinaisons avec clitiques et la négation :

(5)

$\begin{array}{lll}\text { Je lis un livre } & \rightarrow & \text { en lisant un livre } \\ \text { Je ne lis pas de livre } & \rightarrow & \text { en ne lisant pas de livre } \\ \text { Je le lis } & \rightarrow & \text { en le lisant } \\ \text { Je le lui donne } \rightarrow & \text { en le lui donnant } \\ \text { Je ne le lui donne pas } & \rightarrow & \text { en ne le lui donnant pas }\end{array}$

Naturellement, si en remplace un sujet par une marque aspectuelle de devenir métalinguistique du retraitement de cette fonction pour une prédication seconde, ce n'est pas un clitique sujet à proprement parler, comme en témoigne l'impossibilité d'en user en indépendante, et son aspectualité même lui confère des propriétés spécifiques, comme celle d'accepter l'adverbe tout comme marque de perfectivation du traitement 
du sujet en devenir lorsque les procès reliés sont jugés discordants par le locuteur (Halmøy 2003) :

(6) Commandez votre petit déjeuner auprès du service d'étage et appréciez la vue tout en lisant le journal proposé gratuitement chaque matin.

- cas des clitiques ablatif (J'en viens) : en est utilisé lorsque les propriétés $\mathrm{du}$ sujet sont modifiées par l'origine de son mouvement (Je ne vais pas retourner à la cuisine, j'en viens!) ; dans le contraire, on utilise de là (Je viens de Lille / Je viens de là : "j’y suis né », vs j'en viens : «j'arrive de Lille »), où l'origine n'est pas comprise comme un modifieur transitoire des propriétés du sujet à l'instant de parole. Dans ce contexte, en donne à l'interprétant l'instruction d'aspectualiser la relation de l'entité à l'origine du mouvement en considérant la modification courante des propriétés de l'entité imputables au rapport à cette origine.

- cas des clitiques objets (j'en veux (plein / des beaux)) : en remplace un objet partitif ( $d u$ vin, des livres) intégrant la préposition ablative de en fonction d'extracteur occurrentiel relatif à une notion source. Par cette dimension rétrospective, le partitif ne spécifie pas la quantité effectivement obtenue et n'achève pas l'opération de quantification, qui demeure un en-cours métalinguistique: lorsque l'objet partitif est thématisé, c'est en qui le réalise en son site fonctionnel en syntaxe, instruisant l'interprétant de concevoir un objet en cours de définition et qui peut faire l'objet d'un complément de spécification quantitative ou qualitative (j'en veux trois) en réponse à la question de l'inachèvement définitionnel soulevée par en. Le fait que l'objet clitique en soit compatible avec une poursuite de spécification est symptomatique de l'inachèvement et de la cursivité métalinguistique de la constitution de l'objet soulignée en cette position par l'opérateur aspectuel.

On constate donc qu'en français, en, clairement issu de la catégorie de la préposition en diachronie, en est venu à être compatible avec les sites syntaxiques aussi divers que le sujet (d'une prédication retraitée), l'objet, l'introduction d'un syntagme adjectival ou adverbial, et que systématiquement, lorsqu'il survient dans ces positions, il instruit l'interprétant de construire le constituant syntaxique correspondant en l'aspectualisant par la représentation d'une cursivité, d'une transformation ou d'un devenir du même type que celui que -ant flexionnel applique à l'actualisation du temps d'évènement verbal. Ceci rend possible l'hypothèse de l'émergence en français d'un super-opérateur /ã/ muni d'un schéma instructionnel unique, la focalisation d'un en-cours, applicable sous la forme graphique -ant à un déroulement verbal et sous la forme multicatégorielle en aux différentes fonctions syntaxiques que cet opérateur peut assumer. Un tel fonctionnement est évidemment atypique en français et ne semble pas se retrouver chez d'autres marqueurs ; peut-être est-ce précisément parce que en / -ant est sémantiquement en rapport avec la notion de transition et d'instabilité que la classe de mot du signifiant même s'en est trouvée affectée, 
Pré/postpositions, verbes et aspect : trois isomorphismes remarquables en anglais, en français et en basque

déstabilisée et marquée par la multicatégorialité (plutôt que la transcatégorialité, dont on a vu le fonctionnement en basque).

\section{CONCLUSION}

On a relevé dans trois langues trois isomorphismes parallèles liant l'aspectualité de la pré-/postposition à celle de la flexion verbale. Les faits de cohérence morphosyntaxique et sémantique semblent suffisamment concordants pour suggérer que ces convergences ne relèvent pas du hasard ou de l'illusion d'optique même si elles ne s'observent pas dans toutes les langues et ne sauraient être considérées comme des motifs déterministes dans l'évolution de l'organisation d'un système grammatical. Dans notre optique, on dira que les marqueurs grammaticaux fonctionnent comme autant d'activateurs vocaux instruisant l'actualisation de processus cognitifs interprétatifs, et que toute analogie pertinente candidate à l'expérience des usagers du système présente un potentiel de réfection systémique de nature à faire ressortir une régularité, accroître son efficacité et son ergonomie, et aboutir à l'émergence de convergences morphosémantiques du type de in et -ing ou en et -ant, quand elles ne préexistent pas au départ comme dans le cas de la variable $k / n$ en basque. Ce type de modélisation suppose que l'on ne conçoive pas les marqueurs comme des objets qui s'opposeraient toujours rigoureusement : la valeur saussurienne reste un critère d'analyse à un certain niveau, mais il n'est pas exclusif; dès lors que l'on conçoit une forme comme un geste corporel d'amorçage (moteur et sensoriel) susceptible de susciter un effet par semiosis, alors on se trouve dans l'obligation d'interroger systématiquement les faits d'isomorphisme et de considérer leur signification cognitive en matière de procédure de construction du sens.

\section{BIBLIOGRAPHIE}

Achard-Bayle G., 2005, «Polysémie de en: diversité et continuité des changements », in P. Dendale (éd.), Le mouvement dans la langue et la métalangue, Recherches Linguistiques, 27, Metz, 243-270.

Berthoz A. 2009. La simplexité. Odile Jacob.

Bottineau D., 2001, «To entre l'aspect, l'actance et la modalité », P. de Carvalho, N. Quayle, L. Rosier, \& O. Soutet (éds), La psychomécanique aujourd'hui, Actes du $8^{e}$ Colloque international de psychomécanique du langage. Seyssel. 1997., Paris : Champion, France, 49-84.

--- 2003, «Les cognèmes de l'anglais et autres langues », A. Ouattara (éd.), Parcours énonciatifs et parcours interprétatifs, Théories et applications, Actes du Colloque de Troms $\phi$ organisé par le Département de Français de l'Université, 26-28 octobre 2000, Gap : Ophrys, France, 185-201.

--- 2008, «Les formes nominalisées du verbe basque », Faits de Langue, 30, 15 39.

--- 2009, «Les postpositions en basque : quel rapport à la préposition ? », J. François, E. Gilbert, C. Guimier, M. Krause (dir.), Autour de la préposition, 
Bibliothèque de Syntaxe \& Sémantique, Caen : Presses Universitaires de Caen, 91-103.

--- 2010a, Language and enaction, in J. Stewart, O. Gapenne, E. Di Paolo (eds), Enaction: toward a new paradigm for cognitive science, MIT, 267-306.

--- 2010b, «Typologie de la déflexivité », D. Bottineau, L. Begioni (éds.), La déflexivité, Langages, 178, 89-113.

--- 2012, «Les périphrases verbales 'progressives' en anglais, espagnol, français et gallo : aspect, phénoménologie et genèse du sens », C. Bracquenier \& L. Begioni (dir), L'aspect dans les langues naturelles. Approche comparative, Rennes, Presses Universitaires de Rennes, 93-136.

--- 2013, "L'opérateur en en linguistique instructionnelle enactive », Langue française 178, 2/2013 (juin), La préposition en, D. Vigier (éd.), 41-58.

Cadiot P., 2002, "Schémas et motifs en sémantique prépositionnelle : vers une description renouvelée des prépositions dites «spatiales»», Travaux de Linguistique, 44, 1, 9-24.

Cervoni J. 1991. La préposition. Duculot.

Col G., Aptekman J., Girault S. \& Victorri B., 2010, Compositionnalité gestaltiste et construction du sens par instructions dynamiques, Cognitextes 5, Numéro spécial AFLiCo 3 Grammaires en Construction(s), consulté le 01/04/2011 : http://cognitextes.revues.org/372

Dufaye L., 2006, «Localisation spatiale et temporelle avec in, on et at », C. Delmas (éd.), Complétude, cognition, construction linguistique, Presses Sorbonne Nouvelle, 99-109.

Franckel J.-J., Lebaud D. 1991, «Diversité des valeurs et invariance du fonctionnement de en, préposition et préverbe », Langue française, 91, 56-79.

Gaatone D., 1980, «La syntaxe de en et l'obsession de la solution unitaire », Lingvisticae Investigationes, 4, 181-201.

Gilbert E. 2004, «Ébauche d'une formalisation des prépositions in, on et at », J.C. Souesme (éd.), L'identification, Cycnos, 21 (1), 93-111.

Guimier C., 2007, «La préposition et la tradition grammaticale anglaise », F. Neveu, S. Mejri (éds.), La tradition grammaticale, Langages, 167, 85-99.

Halmøy O. 2003. Le gérondif en français. Ophrys.

Hénault C., 2006, "Prépositions en et dans avec certains toponymes : Vers la description d'une variation sémantico-syntaxique dans le français d'aujourd'hui », Les Cahiers de l'AFLS (Association for French Language Studies), vol. 12-1, 2-10.

Menary R., 2010, "Introduction to the special issue on $4 \mathrm{E}$ cognition », Phenomenology and Cognitive Science, 9, 459-463.

Varela F., Thompson E., Rosch E. 1991. The Embodied Mind: Cognitive Science and Human Experience. MIT Press. 